American Journal of Infectious Diseases 8 (1): 65-69, 2012

ISSN 1553-6203

(C) 2012 Science Publications

\title{
Antibiotic Sensitivity Pattern of Blood Isolates of Acinetobacter Species in a Tertiary Care Hospital: A Retrospective Analysis
}

\author{
${ }^{1}$ Shareek, P.S., ${ }^{1}$ D. Sureshkumar, ${ }^{1}$ Ramgopalakrishnan, \\ ${ }^{1}$ V. Ramasubramanian, ${ }^{1}$ K. Abdul Ghafur and ${ }^{2}$ M.A. Thirunarayanan \\ ${ }^{1}$ Department of Infectious Diseases, \\ ${ }^{2}$ Department of Microbiology, \\ Greams lane, Apollo Hospitals, 600006, Chennai, India
}

\begin{abstract}
Problem statement: Multi-drug resistant Acinetobacter bacterium is one of the major causes of sepsis in ICUs in tertiary care hospitals in India. In this report we describe the antibiotic sensitivity patterns of Acinetobacter species isolated from blood over a one year period at a tertiary care hospital. Approach: We retrospectively analyzed the sensitivity pattern of Acinetobacter species isolated from blood during the period $1 / 6 / 2010$ to $31 / 5 / 2011$. Isolation and identification were performed using the best alert system and VITEK2 respectively. Sensitivities were determined by Kirby Bauer disc diffusion and broth dilution using VITEK2 -AST cards. Results: The total number of Acinetobacter species isolated during the study period was 72, out of which $57(79 \%)$ were $A$. baumanii, 7 (9.7\%) were A. Iwofii and $3(5.2 \%)$ were A. Junii. One each from A. calcoaceticus, A. ursingii and $A$. denitrificans were isolated. All of the baumanii isolates were sensitive to polymyxin B and $61.4 \%$ were sensitive to tigecycline. Only $25 \%$ of the isolates in baumanii group were sensitive to meropenem and imipenem. In the non-baumanii group however, $73 \%$ were sensitive to carbapenems. Conclusion: There is a very high incidence of resistance to most antibiotics, including carbapenems. All of the Acinetobacter isolates tested are sensitive to polymyxin B. Tigecycline is the only other drug with reasonable susceptibilities, but this drug is not recommended for primary bacteriemias. If Acinetobacter sepsis is suspected, empiric therapy with polymyxins, followed by de-escalation after sensitivity results are back, is advisable.
\end{abstract}

Key words: Acinetobacter, polymyxin B, colistin, tigecycline, carbapenems

\section{INTRODUCTION}

Members of the genus Acinetobacter are ubiquitous, free living, aerobic, Gram negative coccobacilli that prefer a moist environment and can be easily obtained from soil, water, food and sewage (Dougari 2011).

They are usually considered to be opportunistic pathogens and cause nosocomial infections in hospitalized patients like bacteremia, pneumonia, meningitis and Urinary Tract Infection (UTI) (Towner, 1997; Bergogne-Berezin et al., 1996).

Antimicrobial resistance among nosocomial isolates of Acinetobacter complicates therapy and adversely affects clinical outcomes and treatment costs (Brusselaers et al., 2011; Harris et al., 1999). The presence of resistance to most antibiotic classes requires the use of older and more toxic drugs like colistin for tackling Multi-Drug Resistant (MDR) strains (Fritsche et al., 2005).
We analyzed the resistance pattern of Acinetobacter species grown in blood culture in patients during a one year period.

\section{MATERIALS AND METHODS}

All isolates of Acinetobacter from blood over a period of 1 year $(1 / 6 / 2010$ to $31 / 5 / 2011)$ were included in this study. Isolation and identification was performed using the Bac Talert and the VITEK 2 machine respectively. Antibiotic sensitivities were determined by Kirby Bauer disc diffusion and broth dilution using VITEK2 and AST cards and interpreted according to CLSI criteria.

\section{RESULTS}

The total number of Acinetobacter species isolated during the study period was 72 , out of which 57 (79\%) were A. baumanii, 7 (9.7\%) were A. Iwofii and $3(5.2 \%)$

Corresponding Author: Shareek, P.S., Department of Infectious Diseases, Greams lane, Apollo Hospitals, 600006, Chennai, India 
were A. junii. One each from A. calcoaceticus, A. haemolyticus, A. ursingii, A. denitrificans were isolated. One isolate did not belong to any of the above species.

All the isolates in the Acinetobacter baumanii group were sensitive to polymyxin B (Table 1). 61.4\% were sensitive to tigecycline. Sensitivities of imipenem, meropenem and doripenem were $24.55,24.5$ and $22.5 \%$ respectively. Only $12.5 \%$ of the isolates were sensitive to cefuroxime whereas for cefotaxime and ceftriaxone it was 12.2 and $10.5 \%$ respectively. Cefepime and ceftazidime had marginally better sensitivities when compared with other cephalosporins (28\% each). Cefaperazone + sulbactam combination had better sensitivities $(33 \%)$ when compared to piperacillin + tazobactam $(21 \%)$.

The 15 isolates of non Baumanii group differed significantly from the Baumanii group, which had much greater sensitivities to all classes (Table 2).

Table1: The antibiotic sensitivity pattern of 44 isolates of $A$. baumanii

\begin{tabular}{lll}
\hline Antibiotic & \multicolumn{1}{c}{ Sensitive (\%) } & Intermediate (\%) \\
\hline Polymyxin B & $44(100)$ & \\
Tigecycline & $27(61.4)$ & $15(34.09)$ \\
Cefaperazone/sulbactam & $19(33.3)$ & $4(7)$ \\
Amikacin & $14(24.5)$ & $1(1.75)$ \\
Ciprofloxacin & $16(28)$ & \\
Cotrimoxazole & $13(22.8)$ & \\
Piperacillin/tazobactam & $12(21)$ & \\
Imipenem & $14(24.5)$ & \\
Doripenem & $13(22.8)$ & \\
Meropenem & $14(24.5)$ & \\
Cefuroxime & $7(12.2)$ & \\
Cefotaxime & $7(12.2)$ & \\
Ceftriaxone & $6(10.5)$ & \\
Cefepime & $16(28)$ & \\
Ceftazidime & $16(28)$ & \\
Aztreonam & $8(14)$ & \\
\hline
\end{tabular}

Table 2: Antibiotic sensitivity pattern of 15 isolates of non baumanii group

\begin{tabular}{lll}
\hline Antibiotic & Sensitive (\%) & Intermediate (\%) \\
\hline Polymyxin B & 4 out of 4(100) & \\
Tigecycline & 3out of 4(75) & \\
Cefaperazone+sulbactum & $11(73)$ & $1(6.6)$ \\
GM/Amikacin & $12(80)$ & \\
Cipro/Oflox & $10(66.6)$ & $1(6.6)$ \\
Cotrimoxazole & $7(46.6)$ & \\
Piperacillin+Tazobactam & $11(73)$ & \\
Imipenem & $11(73)$ & \\
Doripenem & $11(73)$ & \\
Meropenem & $11(73)$ & \\
Cefuroxime & $7(46.6)$ & \\
Cefotaxime & $7(46.6)$ & \\
Ceftriaxone & $7(46.6)$ & \\
Cefepime & $12(80)$ & \\
Ceftazidime & $10(66.6)$ & \\
Aztreonam & $7(46.6)$ & \\
\hline
\end{tabular}

\section{DISCUSSION}

Acinetobacter has become one of the most important causes of nosocomial infections (GernerSmidt, 1995; Gulati et al., 1999) and causes considerable mortality as it has acquired many antibiotic resistance genes including the novel carbapenemases. It is an opportunistic pathogen associated with a wide spectrum of infections including nosocomial pneumonia, meningitis, endocarditis, skin and soft tissue infections, urinary tract infections, conjunctivitis, burn wound infections and bacteremia (Bergogne-Berezin and Towner, 1996). The common form of resistance to carbapenems is mediated by lack of drug penetration (i.e., porin mutations and efflux pumps) and/or carbapenem hydrolyzing beta-lactamase enzymes including the Metallo-Betalactamases (MBL). Acquired MBLs are encoded mobile gene cassettes of organism and such strains are often resistant to different groups of antimicrobial agents with transferable properties to various types of bacteria (Pitout et al., 2005).

Resistance rates to carbapenems vary significantly depending on the geographical region. In Greece, the proportion of imipenem-resistant $A$. baumanii isolates from patients hospitalized between 1996 and 2007 in tertiary care hospitals in several regions of the country rose from no resistance to $85 \%$ (ICUs), $60 \%$ (medical wards) and $59 \%$ (surgical wards) GSSAR. Bloodstream isolates from the same dataset exhibited even higher resistance rates. The prevalence of imipenem resistance in $A$. baumanii isolated from a burns unit of USA was found to be as high as $87 \%$ (2007) (Trottier et al., 2007).

Initial Indian studies in the 21 st century showed that Acinetobacter species were fairly sensitive. For instance, Suri et al. (2000) demonstrated Acinetobacter in patients from a neurosurgical unit and it was sensitive to ciprofloxacin, amikacin cefotaxim and ceftriaxone. Singh et al. (2002) showed Acinetobacter which was sensitive to amikacin. Prashanth and Badrinath (2004) from JIPMER Pondichery isolated Acinetobacter which was sensitive to amikacin and ceftazidime. Isolates were resistant to ciprofloxacin and cefotaxime (Prashanth and Badrinath, 2004). Gladstone et al. (2005) from Vellore reported a prevalence of $14 \%$ carbapenem-resistant Acinetobacter spp., isolated from tracheal aspirates.

Banerjee et al. (2005) isolated Acinetobacter from different body fluids which has good sensitivities for gentamycin. Prashanth and Badrinath (2006) showed gradually increasing resistance of Acinetobacter. Gaur et al. (2008) noted resistance to meropenem in $6.4 \%$ of Acinetobacter species. As 
recently as in 2010, one study from Ahmedabad showed few were carbapenem resistant (Patel et al., 2010).

However there are now several Indian studies showing an increased prevalence of MDR Acinetobacter. Our study shows that $75 \%$ of our isolates were carbapenem resistant. This is concordant with recent reports from elsewhere in India. In 2009 a study from Rohtak showed that the resistance of Acinetobacter to meropenem had increased to $25.6 \%$ (Goel et al., 2009). In the same study the resistance to amikacin was $87.2 \%$ and ciprofloxacin was $89.7 \%$. In Delhi, India (2006), the prevalence of carbapenem resistance in Acinetobacter spp. isolated from different clinical samples was found to be almost $35 \%$. Sinha et al. (2007), but the latest studies show resistance to carbapenem is seen in up to $89 \%$ of isolates (Jaggi et al., 2011). In our study the level of carbapenem resistance was very high in the Baumanii group. Karthika et al. (2009) in their study showed the presence of bla IMP1 carbapenemase genes in South Indian population. Though bla VIM-2 is the most common carbapenemases seen in other parts of world (Yum et al., 2002; Poirel et al., 2000; Walsh et al., 2005), it was surprisingly absent in their study, though bla IMP1 gene was seen in $42 \%$ of isolates (Karthika et al., 2009). Our study showed $100 \%$ sensitivity to polymyxin. Tigecycline too retained activity against MDR isolates, although it is not recommended for primary bacteremias. However there have been reports of polymyxin resistant Acinetobacter from Greece, Slovakia and other parts of Europe (Gales et al., 2006; Souli et al., 2006). Polymyxin resistance has been reported from South Korea and the rates of resistance is alarming, 18.1 and $27.9 \%$ for polymyxin $\mathrm{B}$ and colistin respectively (Ko et al., 2007). Recently there has been an alarming study from Chandigarh where $3.5 \%$ of all strains and $16 \%$ of carbapenem resistant strains were resistant to polymyxin B and tigecycline (Taneja et al., 2011).

The antibiotic sensitivity pattern of non Baumanii group differed significantly from Baumanii group. Most of the isolates were sensitive to carbapenems and BL+ BLI combinations (73\%).

The strength of our study is that only blood isolates were analysed, as samples from other sites may represent colonization and may not reuire therapy. The polymyxin group remains the only option as an empirical therapy if Acinetobacter bacteremia is suspected, as it showed $100 \%$ sensitivities to MDR Acinetobacter. Though tigecycline showed good antibiotic sensitivity in our study, it is not recommended for primary bacteremia due to low blood levels resulting inclinical failures.

\section{CONCLUSION}

There was a very high incidence of resistance to most antibiotic classes, including carbapenems, in Acinetobacter blood isolates in our center. All of the resistant isolates were however sensitive to polymyxin B. Tigecycline was the only other drug with reasonable susceptibilities. If Acinetobacter bacteremia is suspected, empiric therapy with the polymyxin group, followed by de-escalation after sensitivity results are back, is advisable.

\section{REFERENCES}

Banerjee, G., M. Singh and N. Goel, 2005. Characterization of Acinetobacter from clinical isolates at Gandhi memorial and associated hospitals, Lucknow. J. Commun. Dis., 37: 30-34. PMID: 16637397

Bergogne-Berezin, E. and K.J. Towner, 1996. Acinetobacter spp. As nosocomial pathogens: microbiological, clinical and epidemiological features. Clin. Microbiol. Rev., 9: 148-165. PMID: 8964033

Bergogne-Berezin, E., M.L. Joly-Guilloo and K.J. Towner, 1996. Acinetobacter: Microbiology, Epidemiology, Infections, Management. 1st Edn., CRC Press, Boca Raton, ISBN: 0849392233, pp: 272.

Brusselaers, N., D. Vogelaers and S. Blot, 2011. The rising problem of antimicrobial resistance in the intensive care unit. Ann. Intensive Care, 1: 47-47. 10.1186/2110-5820-1-47

Dougari, H.J., P.A. Ndakidemi, I.S. Human and S. Benade, 2011. Virulence factors and antibiotic susceptibility among verotoxic non O157: H7 Escherichia coli isolates obtained from water and wastewater samples in Cape Town, South Africa. Afr. J. Biotechnol., 10: 14160-14168.

Fritsche, T.R., H.S. Sader, M.A. Toleman, T.R. Walsh and R.N. Jones, 2005. Emerging metallo-betalactamase-mediated resistances: A summary report from the worldwide SENTRY antimicrobial surveillance program. Clin. Infect. Dis., 41: S276S278. PMID: 16032565

Gales, A.C., R.N. Jones and H.S. Sader, 2006. Global assessment of the antimicrobial activity of polymyxin B against 54731 clinical isolates of Gram-negative bacilli: Report from the SENTRY antimicrobial surveillance programme (20012004). Clin. Microbiol. Infect.,12: 315-321. PMID: 16524407 
Gaur, A., A. Garg, P. Prakash, S. Anupurba and T.M. Mohapatra, 2008. Observations on carbapenem resistance by minimum inhibitory concentration in nosocomial isolates of Acinetobacter species: An experience at a tertiary care hospital in North India. J. Health Popul. Nutr., 26: 183-188. PMID: 18686551

Gerner-Smidt, P., 1995. Taxonomy and epidemiology of Acinetobacter infections. Rev. Med. Microbiol., 6: 186-197.

Gladstone, P., P. Rajendran and K.N. Brahmadathan, 2005. Incidence of carbapenem resistant nonfermenting gram negative bacilli from patients with respiratory infections in the intensive care units. Indian J. Med. Microbiol., 23: 189-191. PMID: 16100428

Goel, N., U. Chaudhary, R. Aggarwal and K. Bala, 2009. Antibiotic sensitivity pattern of gram negative bacilli isolated from the lower respiratory tract of ventilated patients in the intensive care unit. Indian J. Crit. Care Med., 13: 148-151. DOI: 10.4103/0972-5229.58540

Gulati, S., A. Kapil, V. Goel, B. Das and S.N. Dwivedi et al., 1999. Mahapatra AK. Biotyping of Acinetobacter species isolated from clinical samples. Indian J. Med. Res., 110: 160-163. PMID: 10680300

Harris, A., C. Torres-Viera, L. Venkataraman, P. DeGirolami and M. Samore et al., 1999. Epidemiology and clinical outcomes of patients with multiresistant Pseudomonas aeruginosa. Clin. Infect. Dis., 28: 1128-1133. PMID: 10452647

Jaggi, N., P. Sissodia and L. Sharma, 2011. Acinetobacter baumannii isolates: Epidemiology, antibiogram and nosocomial status studied over a 25 month period in a tertiary care hospital in India. Proceedings of the International Conference on Prevention and Infection Control, Jun. 29-Jul. 2, Geneva, Switzerland.

Karthika, R.U., R.S. Rao, S. Sahoo, P. Shashikala and R. Kanungo et al., 2009. Phenotypic and genotypic assays for detecting the prevalence of metallo- $\beta$ lactamases in clinical isolates of Acinetobacter baumannii from a South Indian tertiary care hospital. J. Med. Microbiol., 58: 430-435. DOI: 10.1099/jmm.0.002105-0

Ko, K.S., J.Y. Suh, K.T. Kwon, S.I. Jung and K.H. Park et al., 2007. High rates of resistance to colistin and polymyxin $\mathrm{B}$ in subgroups of Acinetobacter baumannii isolates from Korea. J. Antimicrob. Chemother., 60: 1163-1167. PMID: 17761499
Patel, M.H., G.R. Trivedi, S.M. Patel and M.M. Vegad, 2010. Antibiotic susceptibility pattern in urinary isolates of gram negative bacilli with special reference to AmpC $\beta$-lactamase in a tertiary care hospital. Urol Ann., 2: 7-11. PMID: 20842250

Pitout, J.D., D.B. Gregson, L. Poirel, J.A. McClure and P. Le et al., 2005. Detection of Pseudomonas aeruginosa producing metallo-beta-lactamases in a large centralized laboratory. J. Clin. Microbiol., 43: 3129-3135. PMID: 16000424

Poirel, L., T. Naas, D. Nicolas, L. Collet and S. Bellais et al., 2000. Characterization of VIM-2, a carbapenem-hydrolyzing metallo-beta-lactamase and its plasmid- and integron-borne gene from a Pseudomonas aeruginosa clinical isolate in France. Antimicrob. Agents Chemother, 44: 891-897. PMID: 10722487

Prashanth, K. and S. Badrinath, 2004. In vitro susceptibility pattern of Acinetobacter species to commonly used cephalosporins, quinolones and aminoglycosides. Indian J. Med. Microbiol., 22: 97-103. PMID: 17642704

Prashanth, K. and S. Badrinath, 2006. Nosocomial infections due to Acinetobacter species: Clinical findings, risk and prognostic factors. Indian J. Med. Microbiol., 24: 39-44. PMID: 16505554

Singh, A.K., M.R. Sen, S. Anupurba and P. Bhattacharya, 2002. Antibiotic sensitivity pattern of the bacteria isolated from nosocomial infections in ICU. J. Commun. Dis., 34: 257-63. PMID: 14710856

Sinha, M., H. Srinivasa and R. Macaden, 2007. Antibiotic resistance profile and Extended Spectrum Beta-Lactamase (ESBL) production in Acinetobacter species. Indian J. Med. Res., 63-67. PMID: 17890826

Souli, M., F.V. Kontopidou, E. Koratzanis, A. Antoniadou and E . Giannitsioti et al., 2006. In vitro activity of tigecycline against multiple-drugresistant, including pan-resistant, gram-negative and gram-positive clinical isolates from Greek hospitals. Antimicrob Agents Chemother., 50: 3166-3169. PMID: 16940120

Suri, A., A.K. Mahapatra and A. Kapil, 2000. Acinetobacter infection in neurosurgical intensive care patients. Natl. Med. J. India, 13: 296-300. PMID: 11209484

Taneja, N., G. Singh, M. Singh and M. Sharma, 2011. Emergence of tigecycline and colistin resistant Acinetobacter baumanii in patients with complicated urinary tract infections in north India. Indian J. Med. Res., 133: 681-684. PMID: 21727671 
Towner, K.J., 1997. Clinical importance and antibiotic resistance of Acinetobacter spp. J. Med. Microbiol., 46: 721-746. PMID: 9303951

Trottier, V., P.G. Segura, N. Namias, D. King and L.R. Pizano et al., 2007. Outcomes of Acinetobacter baumannii infection in critically ill burned patients. J. Burn. Care Res., 28: 248-254. PMID: 17351441

Walsh, T.R., M.A. Toleman, L. Poirel and P. Nordmann, 2005. Metallo-beta-lactamases: The quiet before the storm? Clin. Microbiol. Rev., 18: 306-325. PMID: 15831827
Yum, J.H., K. Yi, H. Lee, D. Yong and K. Lee et al., 2002. Molecular characterization of metallo-blactamase-producing Acinetobacter baumannii and Acinetobacter genomospecies 3 from Korea: Identification of two new integrons carrying the blaVIM-2 gene cassettes. J. Antimicrob. Chemother., 49: 837-840. DOI: 10.1093/jac/dkf043 